\title{
A Correlative Model to Predict In Vivo AUC for Nanosystem Drug Delivery with Release Rate-Limited Absorption
}

\author{
Mohammad Barzegar-Jalali ${ }^{1,2}$, Kaivan Mohammadi ${ }^{3}$, Ghobad Mohammadi ${ }^{4}$, Hadi Valizadeh ${ }^{1}$, Azim Barzegar-Jalali ${ }^{5}$ \\ Khosro Adibkia ${ }^{2,6}$, Ali Nokhodchi ${ }^{7}$ \\ ${ }^{1}$ Drug Applied Research Center and ${ }^{2}$ Faculty of Pharmacy, Tabriz University of Medical Sciences, Tabriz, Iran \\ ${ }^{3}$ Mechanical Engineering School, Sharif University of Technology, Tehran, Iran \\ ${ }^{4}$ Faculty of Pharmacy, Kermanshah University of Medical Sciences, Kermanshah, Iran \\ ${ }^{5}$ Faculty of Medicine, Islamic Azad University Ardabil Branch, Ardabil, Iran \\ ${ }^{6}$ Biotechnology Research Center, Tabriz University of Medical Sciences, Tabriz, Iran \\ ${ }^{7}$ Medway School of Pharmacy, Universities of Kent, Chatham, Kent, England
}

Received, July 13, 2012; Revised, October 8, 2012, Accepted, October 11, 2012, Published, October 13, 2012.

\begin{abstract}
Purpose. Drug release from nanosystems at the sites of either absorption or effect biophase is a major determinant of its biological action. Thus, in vitro drug release is of paramount importance in gaining insight for the systems performance in vivo. Methods. A novel in vitro in vivo correlation, IVIVC, model denoted as double reciprocal area method was presented and applied to 19 drugs from 55 nano formulations with total 336 data, gathered from literature. Results. The proposed model correlated the in vitro with in vivo parameters with overall error of $12.4 \pm 3.9 \%$. Also the trained version of the model predicted the test formulations with overall error of $15.8 \pm 3.7 \%$ indicating the suitability of the approach. A theoretical justification was provided for the model considering the unified classical release laws. Conclusion. The model does not necessitate bolus intravenous drug data and seems to be suitable for IVIVC of drugs with release rate-limited absorption.

This article is open to POST-PUBLICATION REVIEW. Registered readers (see "For Readers") may comment by clicking on ABSTRACT on the issue's contents page.
\end{abstract}

\section{INTRODUCTION}

Immense attention has been paid to nanotechnology in various branches of science including medical and pharmaceutical sciences. Numerous research papers in the field of pharmaceutical nanotechnology have been appeared in literature citation. Our focus, however, is on some recently published review articles that deal with drug delivery systems administered via different routes (14). The drug release from pharmaceutical nanosystems is a major determinant in its biological effect. For this reason the release rate of drug from nano delivery system is often measured in vitro to gain insight to its performance in vivo. In the case of various drug delivery systems including immediate and sustained release ones, the correlation between in vitro and in vivo parameters is of paramount importance. The United States
Pharmacopeia discusses the importance as well as different levels of the correlation (5). Also the details and advantages of such correlations were reviewed in a recent article (6). One of the important advantages of establishing in vitro- in vivo correlation, IVIVC, is the calculation of in vivo parameter from the correlation equation after carrying out only in vitro test without performing in vivo experiment on the drug product under consideration $(5,6)$. Despite extensive investigations on drug nanosystems from in vitro and in vivo points of view, to the best of our knowledge no comprehensive IVIVC study was reported in literature. In the present work the available in vitro release as well as in vivo data of drug nanosystems gathered from various sources was subjected to a

Corresponding Author: Ghobad Mohammadi; Kermanshah University of Medical Sciences, Daneshgah Ave.,

Kermanshah, Iran.ghmohammadi@kums.ac.ir 
previously empirical novel IVIVC approach named double reciprocal area, DRA, method $(7,8)$.The area under drug plasma concentration or biological response vs. time curve, $\mathrm{AUC}_{\mathrm{i}}$ and the area under drug release curve vs. time were employed as in vivo and in vitro parameters for correlation purpose. The method predicted in vivo parameter from corresponding in vitro data with good accuracy and precision. Unlike the previously established IVIVC methods, the proposed model is less invasive because it does not involve bolus intravenous data of drug. A theoretical justification was also provided for the empirical method.

\section{METHODS}

Data

The in vitro and in vivo data of 17 drugs from 55 nanosystems with total number of 336 data points were collected from literature. The name of drugs and the relevant references are given in Table 1 (9-27). As seen in the table the data includes several kind of nanosystems intended for administration via different routes. The coordinates of each point in the in vitro and in vivo profiles of drug nanosystems in the original papers were measured carefully and the data was employed in the subsequent IVIVC analysis.

\begin{tabular}{llllllllllll}
\hline \multicolumn{1}{l}{ Table 1. Details of drugs, nanosystems, statistical parameters and constants of double reciprocal area IVIVC model. } \\
\hline No. & Drug & NF & N & TNS & R & EC & RA & m & b & j & Ref. \\
\hline $\mathbf{1}$ & 5-Fluorouracil* & 3 & 21 & NS & 0.725 & 19.2 & p.o & 28.419 & 0.980 & 0.901 & $(9)$ \\
$\mathbf{2}$ & Carbazole & 2 & 10 & CL-NP & 0.983 & 7.5 & p.o & 4.048 & 0.954 & 0.021 & $(10)$ \\
$\mathbf{3}$ & Celecoxib & 3 & 24 & SLH & 0.980 & 7.2 & p.o & 303.8 & 1.271 & 1.151 & $(11)$ \\
$\mathbf{4}$ & Danazol & 3 & 18 & NS & 0.889 & 16.6 & p.o & 0.114 & 0.637 & 0.002 & $(12)$ \\
$\mathbf{5}$ & Docetaxel & 4 & 24 & NM & 0.956 & 13.8 & p.o & 0.005 & 0.796 & 0.000 & $(13)$ \\
$\mathbf{6}$ & Estradiol** & 2 & 12 & NM & 0.970 & 13.2 & p.o & 0.755 & 0.602 & 0.000 & $(14)$ \\
$\mathbf{7}$ & Estradiol** & 3 & 18 & NM & 0.929 & 12.4 & p.o & 0.455 & 0.900 & 0.02 & $(15)$ \\
$\mathbf{8}$ & Estradiol** & 4 & 24 & NM & 0.804 & 8.1 & p.o & 0.505 & 1.185 & 0.058 & $(16)$ \\
$\mathbf{9}$ & Flurbiprofen & 2 & 12 & LN & 0.999 & 16.7 & p.o & 0.687 & 1.196 & 0.015 & $(17)$ \\
& & & & & & & & & & & \\
$\mathbf{1 0}$ & Heparin*** & 3 & 18 & NS & 0.936 & 9.70 & p.o & 0.567 & 1.182 & 0.050 & $(18)$ \\
$\mathbf{1 1}$ & Insulin & 4 & 24 & NMA & 0.905 & 11.8 & n.s & 0.444 & 1.185 & 0.016 & $(19)$ \\
$\mathbf{1 2}$ & Methotrexate & 4 & 24 & SLN & 0.958 & 10.5 & p.o & 3.057 & 1.312 & 0.333 & $(20)$ \\
$\mathbf{1 3}$ & Mifepristone & 2 & 16 & NS & 0.756 & 15.9 & p.o & 0.043 & 1.18 & 0.004 & $(21)$ \\
$\mathbf{1 4}$ & Nifedipine & 3 & 18 & NM & 0.967 & 8.6 & p.o & 0.028 & 0.806 & 0.001 & $(22)$ \\
$\mathbf{1 5}$ & Nitrendipine & 3 & 18 & SLN & 0.826 & 8.2 & i.d & 0.008 & 1.019 & 0.006 & $(23)$ \\
$\mathbf{1 6}$ & Rhodamine & 2 & 10 & NM & 0.758 & 18.2 & p.o & 0.458 & 0.907 & 0.442 & $(24)$ \\
& (Model dye) & & & & & & & & & & \\
$\mathbf{1 7}$ & Tobramycin & 3 & 18 & SLN & 0.831 & 16.53 & i.d & 0.052 & 0.721 & 0.032 & $(25)$ \\
$\mathbf{1 8}$ & Ucb & 2 & 12 & NC & 0.957 & 13.1 & p.o & 0.137 & 0.701 & 0.001 & $(26)$ \\
& (Anti asthma) & & & & & & & & & & \\
$\mathbf{1 9}$ & Vinpocetine & 3 & 15 & SLN & 0.923 & 8.5 & p.o & 3.208 & 0.783 & 0.311 & $(27)$ \\
\hline
\end{tabular}

$\mathrm{NF}$, number of formulations for a given drug; N, number of data for each drug; TNS, type of nanosystems; R, correlation coefficient; EC, error of correlation in percentage; RA, route of administration; $\mathrm{m}, \mathrm{b}$ and $\mathrm{j}$, model constants; NS, nanosphere; CL-NP, cross-linked nanoparticle; SLH, silica-lipid hybrid particle; NM, nanomatrix; LN, lipid nanoparticle; NMA, nano mucoadhesive; SLN, solid lipid nanoparticle; NC, nano crystal; p.o, per oral; n.s, nasal; i.d, intraduodenal. *One of the formulations of 5-Fluorouracil was the pure drug itself the inclusion of which improved the accuracy of the model. ** The difference in the molecular weight of polymer and solvents involved in the formulations as well as the particle size of the nanosystems affected the model parameters of the same drug estradiol. ***In the case of heparin the biological response was used. The overall mean error, OEC, for 19 drugs calculated by equation 3 is $12.4 \pm 3.9 \%$. 
Double reciprocal area IVIVC method

The double reciprocal area, DRA, model applied successfully for IVIVC $(7,8)$ has been advocated to establish a quantitative correlation between in vitro and in vivo parameters of all drug nanosystems given in Table 1. In addition to the total AUC, the partial AUC is also used in bioavailability studies $(5,8)$. The following nonlinear relationship represents the model:

$$
\frac{1}{\left(A U C_{i}\right)_{0}^{t_{n}}}=m\left(\frac{1}{\left[\left(A U C_{r}\right)_{0}^{t_{n}}\right]^{b}}\right)+j
$$

Where $\left(A U C_{i}\right)_{0}^{t_{n}}$ is the area under the drug plasma concentration or biological responses $v s$ normalized time $\left(\mathrm{t}_{\mathrm{n}}\right)$ curve between 0 and $\mathrm{t}_{\mathrm{n}}$ $;\left(A U C_{r}\right)_{0}^{t_{n}}$ is area under percent drug released $v s \mathrm{t}_{\mathrm{n}}$ curve between 0 and $\mathrm{t}_{\mathrm{n}}$. These areas are observed ones. The symbols $\mathrm{m}, \mathrm{b}$ and $\mathrm{j}$ are model constants. Several factors are involved in drug release from the complex nanosystems. These include nature of drug, method of preparation and excipients of formulation, technique of release study and mechanisms of processes affecting the overall release. Some of the processes in the release from nanosystems are wettability, dispersability and penetration of water molecules into the nanosystem; hydration and swelling of the polymer in the system as well as drug dissolution and diffusion. These factors which complicate the overall drug release process reflect in the mentioned parameters. Thus, the exact nature of the parameters cannot be clarified. The reason for the use of $\mathrm{AUC}_{\mathrm{i}}$ in the correlation is based on well-known pharmacokinetic principles according which in vivo AUC of drug is directly related to its extent of bioavailability and the latter is related to clinical response.

The normalized time is the ratio of any in vivo and in vitro sampling time with respect to the corresponding arbitrary last sampling time. The normalization of time is necessary to bring in vivo and in vitro times to the same scale. The percent released corresponding to each in vivo value of $t_{n}$ was obtained by interpolation of the normalized release curve. The values of $\left(A U C_{i}\right)_{0}^{t_{n}}$ and $\left(A U C_{r}\right)_{0}^{t_{n}}$ were calculated via trapezoidal rule. The left side of equation 1 was regressed nonlinearly against $\frac{1}{\left[\left(A U C_{r}\right)_{0}^{t_{n}}\right]^{b}}$ to obtain numerical values of $\mathrm{m}, \mathrm{b}$ and $\mathrm{j}$ of the model. The correlation error, EC, for nanosystems of individual drug was calculated by:

$$
E C=\frac{100}{N} \times \sum_{1}^{N} \frac{\left|\left[\left(A U C_{i}\right)_{0}^{t_{n}}\right]_{c a l}-\left[\left(A U C_{i}\right)_{0}^{t_{n}}\right]_{o b s}\right|}{\left[\left(A U C_{i}\right)_{0}^{t_{n}}\right]_{o b s}}
$$

$N,\left[\left(A U C_{i}\right)_{0}^{t_{n}}\right]_{\text {cal }}$ and $\left[\left(A U C_{i}\right)_{0}^{t_{n}}\right]_{o b s}$ are number of data which belongs to any drug, calculated $\left(A U C_{i}\right)_{0}^{t_{n}}$ by the model and observed $\left(A U C_{i}\right)_{0}^{t_{n}}$, respectively. The calculated $\mathrm{AUC}_{\mathrm{i}}$ is obtained after insertion of observed $\mathrm{AUC}_{\mathrm{r}}$, numerical values of $\mathrm{m}, \mathrm{b}$ and $\mathrm{j}$ into the correlation equation 1 . The overall mean error of correlation, OEC, for 19 drugs in table 1 was also calculated using equation 3:

$$
O E C=\sum_{1}^{19} \frac{E C}{19}
$$

\section{Development of the IVIVC model}

The rate of drug release from nanosystems and solid dispersions in terms of amounts released, w, and unreleased ,M, was described by an equation obtained from unification of the Noyes- Whitney law of dissolution and Flick's first law in diffusion with introduction of a time dependent variable $\mathrm{X}(28,29)$ :

$$
\frac{d w}{d t}=-\frac{d M}{d t}=\frac{D}{h} \mathrm{SC}_{\mathrm{s}} \mathrm{X}
$$

In which w is amount of drug released up to time t. $d w / d t$ is the rate of release in term of $w$. The symbols $D, S, C_{s}$ and $h$ are drug 
molecule diffusion coefficient, effective surface area of drug, drug solubility in the medium and the length of diffusion path, respectively $(28,29)$. In context of drug nanosystems the release medium can be in vitro simulation of either the absorption site or the action biophase e.g. inside of cells after endocytosis. The value of $\mathrm{X}$ represents all time-dependent variables affecting drug release in the simulated medium. For a complex system such as nanoparticles, the classical equations of the drug release mentioned above do not include factors influencing the drug release rate among which penetration rate of liquid into the system; hydration, swelling, relaxation, erosion and dissolution of polymer can be mentioned. The extents of liquid penetration and the polymer contributed properties are directly proportional to $t_{1 / 2}$ and powered of $t$, respectively all of which are embedded in variable $X(28,29)$. Therefore, assuming all parameters with the exception of $\mathrm{C}_{\mathrm{s}}$ in the right-hand site of equation 4 are timedependent, its integration between times 0 and $t$ gives:

$$
\begin{gathered}
w=C_{S} \int_{0}^{t}\left(\frac{D S X}{h}\right) d t \\
M=M_{0}-C_{S} \int_{0}^{t}\left(\frac{D S X}{h}\right) d t
\end{gathered}
$$

$\mathrm{M}_{0}$ is the amount of drug in nanosystem at time 0 . Since the value of $\mathrm{w}$ increases with time the integral term in equations 5 and 6 should increase with time as well. Also, M deceases with time. The closest measurable substitute supported by experimental evidence for the integral term in equations 5 and 6 is $\int_{0}^{t} w \cdot d t$, area under the release curve between times 0 and $t,\left(A U C_{r}\right)_{0}^{t}(7,8)$. The latter parameter represents better the cumulative changes of time- dependent variables embedded in the equations at absorption and the biophase sites. Thus, w can be expressed in term of the latter area as power equation 7 :

$$
w=C_{s} \beta\left[\left(A U C_{r}\right)_{0}^{t}\right]^{\alpha}
$$

The symbols $\alpha$ and $\beta$ are constants. The negative sign preceding the integral term of the equation 6 indicates an inverse relationship between $\mathrm{M}$ and the integral term. The difference between constant $\mathrm{M}_{0}$ and the integral term can be approximated by an inverse powered integral term in the form of:

$$
M=\beta^{\prime}\left[\left(A U C_{r}\right)_{0}^{t}\right]^{-\alpha^{\prime}}
$$

The values of $\alpha^{\prime}$ and $\beta^{\prime}$ are constants. The positive $\alpha$ and negative $\alpha^{\prime}$ indicate that $w$ increases and $M$ decreases with time. Thus, equation 8 reflects explicitly the inverse relation between $\mathrm{w}$ and $\mathrm{M}$. Similar power relationships for $\mathrm{w}$ and $\mathrm{M}$ were given in previous papers $(28,29)$. Fraction of drug released, F, up to any time is given by:

$$
F=\frac{w}{w+M}=\frac{C_{S} \beta\left[\left(A U C_{r}\right)_{0}^{t}\right]^{\alpha}}{C_{S} \beta\left[\left(A U C_{r}\right)_{0}^{t}\right]^{\alpha}+\beta^{\prime}\left[\left(A U C_{r}\right)_{0}^{t}\right]^{-\alpha^{\prime}}}=\frac{C_{S} \beta\left[\left(A U C_{r}\right)_{0}^{t}\right]^{\alpha+\alpha^{\prime}}}{C_{S} \beta\left[\left(A U C_{r}\right)_{0}^{t}\right]^{\alpha+\alpha^{\prime}}+\beta^{\prime}}=\frac{\left[\left(A U C_{r}\right)_{0}^{t}\right]^{b}}{\left[\left(A U C_{r}\right)_{0}^{t}\right]^{b}+\mu}
$$

Where $b=\alpha+\alpha$ ', and $\mu=\beta^{\prime} / \beta C_{s}$. For establishing a meaningful correlation between in vitro and in vivo parameters it is logical to use area under the curve of biological response and/or plasma drug level, $\left(A U C_{i}\right)_{0}^{t}$ as an in vivo parameter $(7,8)$. It is worth to 
mention that the partial area under the in vivo curve, $\left(A U C_{i}\right)_{0}^{t}$, has been recommended by USP as a measure of extent of drug bioavailability. It is obvious in drug release rate- limited biological response and/ or bioavailability there must be a quantitative correlation between $\left(A U C_{i}\right)_{0}^{t}$ and $F$. It would be reasonable to assume a direct relation between the latter parameters via introducing a proportionality constant $k$ :

$$
\left(A U C_{i}\right)_{0}^{t}=k F=\frac{k\left[\left(A U C_{r}\right)_{0}^{t}\right]^{b}}{\left[\left(A U C_{r}\right)_{0}^{t}\right]^{b}+\mu}
$$

Reciprocating equation 10 and subsequent substitution of corresponding normalized areas and rearrangement yields the double reciprocal area method of IVIVC, equation 1, in which $m=\mu / k$ and $j=1 / k$.

\section{Training DRA model for prediction}

The aim of obtaining trained model was to determine model parameters for each drug and use the parameters for estimating $\mathrm{AUC}_{\mathrm{i}}$ of an excluded formulation of the same drug from training procedure. To this end, 13 out of 19 drugs (Table 2) with 3 and 4 formulations altogether 264 data were used to assess prediction ability of the model. The drugs with two formulations were omitted from the prediction analysis because of statistical limitation. In the case of drug with 3 formulations, 3 possible combinations consisted of in vitro and in vivo data of formulations 1,$2 ; 1,3$ and 2,3 were employed for training of the model to obtain the numerical values of the model constants. It is obvious that in the training process formulations 3, 2 and 1 were excluded from the combinations, respectively. Then, after inserting the in vitro parameter of the excluded formulation into the trained model, it's in vivo parameter was predicted. The same procedure was followed for drugs with 4 formulations. That is 4 possible combinations each consisted of 3 formulations for prediction of the in vivo parameters of the excluded ones. The prediction error, $P E$, for excluded formulation of each drug is given by:

$P E=\frac{100}{q} \sum_{1}^{q} \frac{\left|\left[\left(A U C_{i}\right)_{0}^{t_{n}}\right]_{p r e d}-\left[\left(A U C_{i}\right)_{0}^{t_{n}}\right]_{o b s}\right|}{\left[\left(A U C_{i}\right)_{0}^{t_{n}}\right]_{o b s}}$

In which $\mathrm{q}$ is the number of in vivo data for excluded formulation of individual drug and $\left[\left(A U C_{i}\right)_{0}^{t_{n}}\right]_{\text {pred }}$ is its predicted area under the curve by means of the trained model. The accuracy of the trained models for each drug was evaluated by calculating mean prediction error, MPE:

$$
M P E=\frac{1}{N} \sum_{1}^{N} P E
$$

$\mathrm{N}$ is 3 and 4 for drugs with 3 and 4 formulations respectively. Also the overall prediction error, OMPE, was assessed taking the average of MPEs for 13 drugs in Table 2.

\section{RESULTS}

Table 1 shows the results of in vitro- in vivo correlation by DRA model. All the correlation errors were below $20 \%, 68.4 \%$ of ECs were below $15 \%$ and $36.8 \%$ were less than $10 \%$. The overall error of correlation, OEC, was $12.4 \pm 3.9 \%$. The minimum and maximum ECs were $7.2 \%$ and $19.2 \%$, respectively. The values of $\mathrm{R}$ indicated that the correlations were highly significant ( $p$ levels between 0.001 and 0.0005 ). The model parameters $m$, $b$ and $j$ were also highly significant at the mentioned levels. The model parameters $m, b$ and $j$ were also highly significant. The level of significance for the parameters was generally at $\mathrm{p}$ values less than 0.001 . The correlation between $\left[\left(A U C_{i}\right)_{0}^{t_{n}}\right]_{o b s}$ and $\left[\left(A U C_{i}\right)_{0}^{t_{n}}\right]_{c a l}$ for 336 data is depicted in Fig. 1. 
Table 2. Drugs with 3 and 4 nanosystem formulations for training, assessing prediction ability of DRA model and its mean prediction error, MPE together with overall mean prediction error, OMPE.

\begin{tabular}{llllll}
\hline No. & Drug & MPE & No. & Drug & MPE \\
\hline $\mathbf{1}$ & 5-Fluorouracil & 22.7 & 8 & Insulin & 17.6 \\
$\mathbf{2}$ & Celecoxib & 12.4 & 9 & Methotrexate & 10.0 \\
$\mathbf{3}$ & Danazol & 15.2 & 10 & Nifedipine & 14.7 \\
$\mathbf{4}$ & Docetaxel & 16.1 & 11 & Nitrendipine & 13.9 \\
$\mathbf{5}$ & Estradiol & 14.1 & 12 & Tobramycin & 22.5 \\
$\mathbf{6}$ & Estradiol & 12.7 & 13 & Vinpocetine & 16.1 \\
$\mathbf{7}$ & Heparin* & 17.4 & OMPE & $15.8 \pm 3.7$ \\
\hline *In the case of heparin the biological response was used. \\
\hline
\end{tabular}

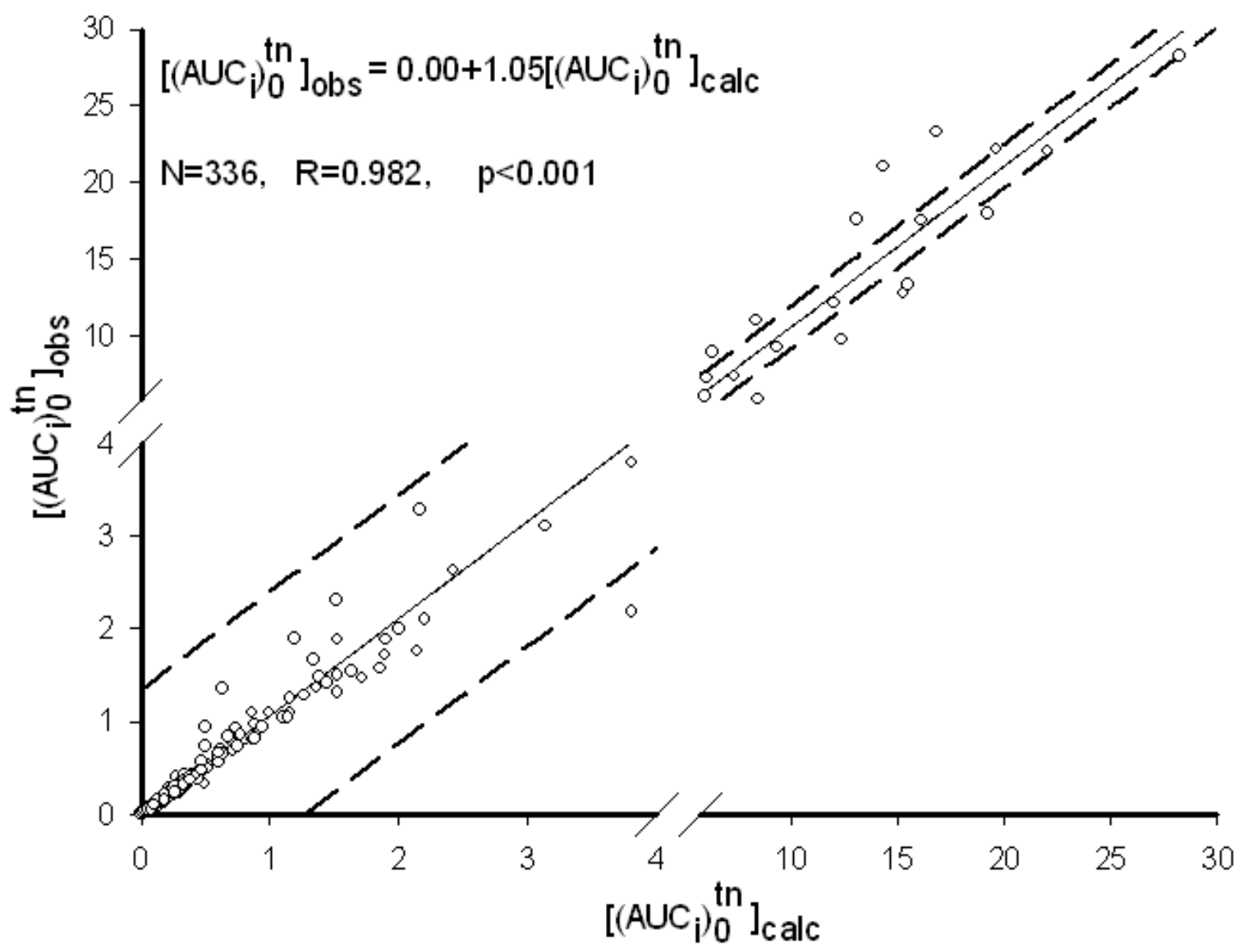

Figure 1. Observed area under the drug plasma concentration or biological response curve, $\left[\left(\boldsymbol{A U} \boldsymbol{C}_{\boldsymbol{i}}\right)_{\mathbf{0}}^{\boldsymbol{t}_{\boldsymbol{n}}}\right]_{\boldsymbol{o b s}}$, against the calculated area, $\left[\left(\boldsymbol{A} \boldsymbol{U} \boldsymbol{C}_{\boldsymbol{i}}\right)_{\mathbf{0}}^{\boldsymbol{t}_{\boldsymbol{n}}}\right]_{\boldsymbol{c} \boldsymbol{c} \boldsymbol{c} \boldsymbol{c}}$ based on the proposed novel IVIVC model. The upper and lower 95\% prediction interval is represented by the broken lines. For the sake of clarity in construction of the regression line, all drug plasma concentrations were expressed in $\mu \mathrm{g} / \mathrm{ml}$.

When the trained models were applied to the excluded formulations the minimum mean prediction error, MPE, was $10 \%$ and the maximum was $22.7 \%$. Overall mean prediction error, OMPE, was $15.8 \pm 3.7 \%$.
The errors of 11 out of 13 drugs were below $20 \%$ (Table 2). In Fig. 2 the plot of $\left[\left(A U C_{i}\right)_{0}^{t_{n}}\right]_{o b s}$ vs $\left[\left(A U C_{i}\right)_{0}^{t_{n}}\right]_{\text {pred }}$ is seen for 264 data. 


\section{DISCUSSION}

It is evident from the tables and figures that the proposed model can correlate not only very well the in vitro with in vivo data but also the prediction capability and reproducibility of its trained versions is quite acceptable. In the correlation procedure $96.7 \%$ of 336 data are in $95 \%$ prediction interval (Fig. 1). Also $95.8 \%$ of the 264 predicted values from the trained models are in the same prediction interval (Fig. 2). These findings indicate the suitability of the proposed model.

This approach of IVIVC can be considered as a novel point by point method (similar to level A correlation in USP). Similar to level A correlation in USP the novel proposed model is a point by point deconvolution method. Unlike most other deconvolution approaches, the model requires no intravenous data. In the USP level A approach, the relationship between the fraction of drug absorbed up to any time and percent drug released in vitro up to the same time is linear (8). However, since the present novel DRA model employs the partial area in place of the fraction absorbed, it correlates with the fraction absorbed in a nonlinear fashion (equation 1). The partial area is dependent not only on the drug release rate but also is affected by drug clearance from the body. Therefore, for drugs exhibiting release rate limited absorption from nanosystems, the area should depend highly on the release rate at the absorption site (8). Due to profound effect of release and/or fraction absorbed it is expected that $A U C_{i}$ relates to release, but because of the interference of the opposing effect of clearance the IVIVC profile is nonlinear. The OEC and OMPE of the novel DRA model and its trained versions are $12.4 \pm 3.9 \%$ and $15.8 \pm 3.7 \%$, respectively which are quite acceptable considering the inherent error associated with integration process employing the trapezoidal rule.

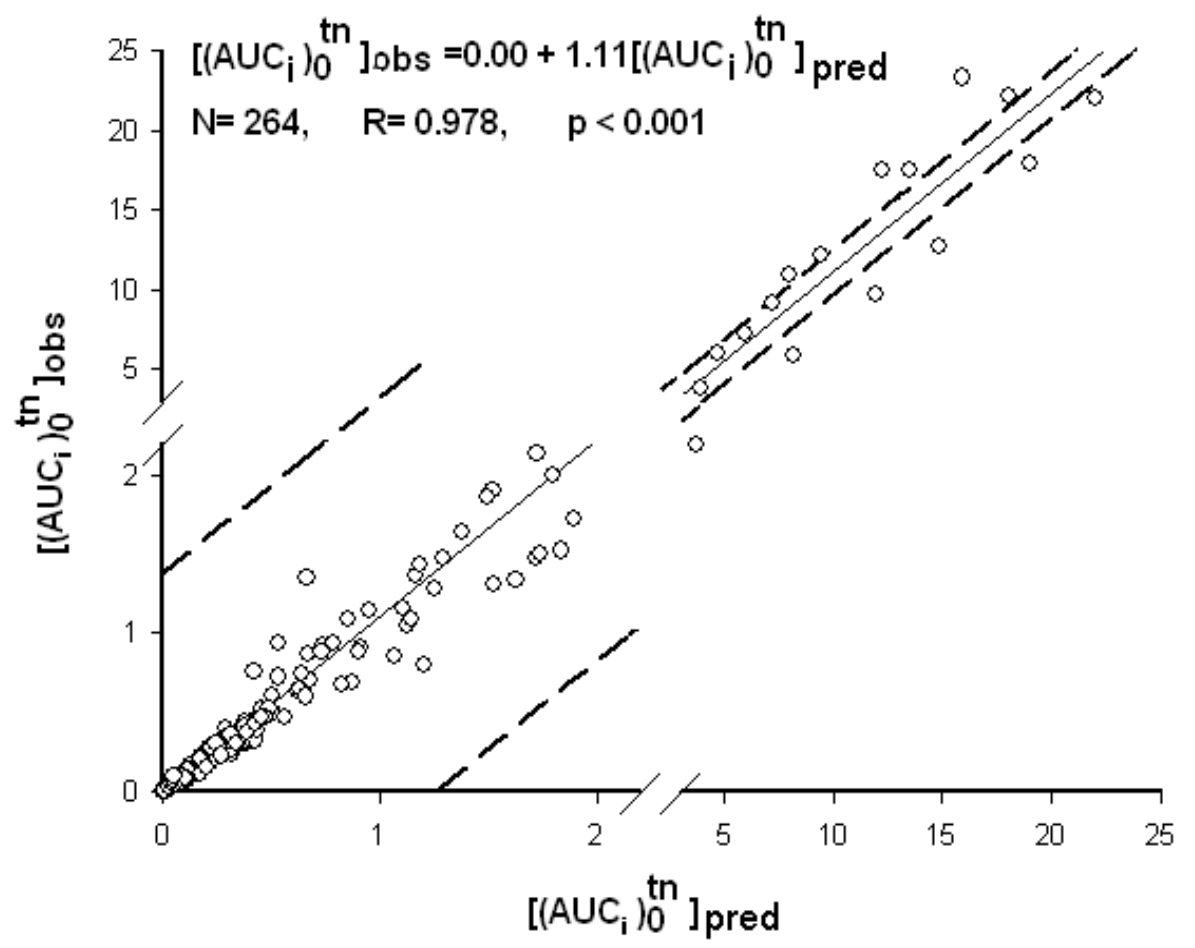

Figure 2. Observed area under the drug plasma concentration or biological response curve, $\left[\left(\boldsymbol{A U} \boldsymbol{C}_{\boldsymbol{i}}\right)_{\mathbf{0}}^{\boldsymbol{t}_{\boldsymbol{n}}}\right]_{\boldsymbol{o b s}}$, against the predicted area, $\left[\left(\boldsymbol{A} \boldsymbol{U} \boldsymbol{C}_{\boldsymbol{i}}\right)_{\mathbf{0}}^{\boldsymbol{t}_{\boldsymbol{n}}}\right]_{\text {pred }}$ based on the trained proposed novel IVIVC model. The upper and lower $95 \%$ prediction interval is represented by the broken lines. For the sake of clarity,in the construction of the regression line, all drug plasma concentrations were expressed in $\mu \mathrm{g} / \mathrm{ml}$. 


\section{CONCLUSION}

A kind of novel point by point nonlinear IVIVC model was presented and applied to 17 drugs in 55 nanosystems successfully with overall error of correlation $12.4 \%$. A theoretical justification for the model was also provided. When the trained versions of the model were used for prediction of in vivo parameter of untested formulations, the overall error was satisfactory (15.8\%). Thus the model could be employed as a suitable IVIVC approach for drug nanosystems. The model may be suitable for IVIVC of drugs with release rate-limited absorption. It may have also advantage over the conventional methods in that it does not require drug data from bolus intravenous drug injection.

\section{ACKNOWLEDGMENT}

We would like to thank Drug Applied Research Center, Tabriz University of Medical Sciences, Tabriz, Iran for the financial support of this project.

\section{REFERENCES}

1. Mishra, B., Patel, B.B., Tiwari, S. Colloidal nanocarriers: a review on formulation technology, types and applications toward targeted drug delivery. Nanomed-Nanotechnol, 6 (1), 9-24, 2010.

2. Zhao, Y., Brown, M.B., Jones, S.A. Pharmaceutical foams: are they the answer to the dilemma of topical nanoparticles? NanomedNanotechnol, 6 (2), 227-236, 2010.

3. Cervin, C., Tinzl, M., Johnsson, M., Abrahamsson, PA., Tiberg, F., Dizeyi, N. Properties and effects of a novel liquid crystal nanoparticle formulation of docetaxel in a prostate cancer mouse model. Europ J Pharm Sci, 41 (2), 369-375, 2010.

4. Chen, MC.,Sonaje,K., Chen, KJ., Sung, HW. A review of the prospects for polymeric nanoparticle platforms in oral insulin delivery. Biomaterials, 32 (36), 9826-9838, 2011.

5. The United States Pharmacopeia, The National Formulary; USP31, NF26. The United States Pharmacopeial Convention, Rockville, MD, pp. 553-557, 2008.

6. Emami, J. In vitro - In vivo Correlation: From Theory to Applications. J Pharm Pharm Sci, 9, 169-189, 2006.
7. Dastmalchi, S., Garjani, A., Maleki, N., Sheikhee, G., Baghchevan, V., Jafari-Azad, P., Valizadeh, H., Barzegar-Jalali, M., 2005.Enhancing dissolution, serum concentrations and hypoglycemic effect of glibenclamide using solvent deposition technique. J Pharm Pharm Sci, 8, 175-181, 2005.

8. Barzegar-Jalali, M., Mohajjel Nayebi, A., Valizadeh, H., Hanaee, J., Barzegar-Jalali, A., Adibkia, K., Anoush, M., Sistanizad, M. Evaluation of in vitro-in vivo correlation and anticonvulsive effect of carbamazepine after cogrinding with microcrystalline cellulose. J Pharm Pharm Sci, 9, 307-316, 2006.

9. Zheng, Y., Yang, W., Wang, C., Hu, J., Fu, S., Dong, L., Wu, L., Shen, X. Nanoparticles based on the complex of chitosan and polyaspartic acid sodium salt: Preparation, characterization and the use for 5-fluorouracil delivery. Europ J Pharm Biopharm, 67, 621-631, 2007.

10. Arangoa, MA.,Campanero, MA., Renedo, MJ., Ponchel, G., Irache, JM. Gliadin Nanoparticles as Carriers for the Oral Administration of Lipophilic Drugs. Relationships between Bioadhesion and Pharmacokinetics. Pharm Res,18, 1521-1527, 2001.

11. Tan, A., Simovic, S., Davey, AK., Rades, T., Prestidge, CA. Silica-lipid hybrid (SLH) microcapsules: A novel oral delivery system for poorly soluble drugs. J Control Release, 134, 6270, 2009.

12. Purvis, T., Vaughn, JM., Rogers, TL., Chen, X., Overhoff, KA., Sinswat, P., Hu, J., McConville, JT., Johnston, KP., Williams, RO. Cryogenic liquids, nanoparticles and microencapsulation. Int J Pharm, 324, 43-50, 2006.

13. Feng, SS., Mei, L., Anitha, P., Gan, CW., Zhou, W. Poly (lactide)-vitamin E derivative/montmorillonite nanoparticle formulations for the oral delivery of Docetaxel. Biomaterials, 30, 3297-3306, 2009.

14. Hariharan, S., Bhardwaj, V., Bala, I., Sitterberg, J., Bakowsky, U., Ravi Kumar, MNV. Design of Estradiol Loaded PLGA Nanoparticulate Formulations: A Potential Oral Delivery System for Hormone Therapy. Pharm Res, 23, 184-195, 2006.

15. Sahana, DK., Mittal, G., Bhardwaj, V., Ravi Kumar, M. PLGA nanoparticles for oral delivery of hydrophobic drugs: Influence of organic solvent on nanoparticle formation and release behavior in vitro and in vivo using estradiol as a model drug. J Pharm Sci, 97, 1530-1542, 2008.

16. Mittal, G., Sahana, DK., Bhardwaj, V., Ravi Kumar, M. Estradiol loaded PLGA nanoparticles for oral administration: Effect of polymer molecular weight and copolymer composition on release behavior in vitro and in vivo. J Control Release, 119, 77-85, 2007.

17. Bhaskar, K., Anbu, J., Ravichandiran, V., Venkateswarlu, V., Rao, YM. Lipid nanoparticles 
for transdermal delivery of flurbiprofen: formulation, in vitro, ex vivo and in vivo studies. Lipids Health Dis, 8, 1-8, 2009.

18. Jiao, Y., Ubrich, N., Marchand-Arvier, M., Vigneron, C., Hoffman, M., Lecompte, T., Maincent, $\mathrm{P}$. In vitro and in vivo evaluation of oral heparin-loaded polymeric nanoparticles in rabbits. Circulation, 105, 230-235, 2002.

19. Jain, AK.,Khar, RK., Ahmed, FJ., Diwan, PV. Effective insulin delivery using starch nanoparticles as a potential trans-nasal mucoadhesive carrier. Europ J Pharm Biopharm, 69, 426-435, 2008.

20. Paliwal, R., Rai, S., Vaidya, B., Khatri, K., Goyal, AK., Mishra, N., Mehta, A., Vyas, P. Effect of lipid core material on characteristics of solid lipid nanoparticles designed for oral lymphatic delivery. Nanomed-Nanotechnol, 5, 184-191, 2009.

21. He, W., Horn, SW., Hussain., MD. Improved bioavailability of orally administered mifepristone from PLGA nanoparticles. Int J Pharm, 334, 173178, 2007.

22. Kim, Y., Fluckiger, L., M. Hoffman,I. LartaudIdjouadiene,J. Atkinson,P. M. The antihypertensive effect of orally administered nifedipine-loaded nanoparticles in spontaneously hypertensive rats. Brit J Pharmacol, 120, 399-404, 1997.

23. Kumar, VV., Chandrasekar, D., Ramakrishna, S., Kishan, V., Rao, YM.,Diwan, PV. Development and evaluation of nitrendipine loaded solid lipid nanoparticles: Influence of wax and glyceride lipids on plasma pharmacokinetics. Int J Pharm, 335, 167-175, 2007.
24. Samstein, RM., Perica, K., Balderrama, F., Look, M., Fahmy, TM. The use of deoxycholic acid to enhance the oral bioavailability of biodegradable nanoparticles. Biomaterials, 29, 703-708, 2008.

25. Cavalli, R., Bargoni, R., Podio, V., Buntoni, E., Zara, GP.,Gasco, MR. Duodenal administration of solid lipid nanoparticles loaded with different percentages of tobramycin. J Pharm Sci, 92, 10851094, 2003.

26. Hecq, J., Deleers, M., Fanara, D., Vranckx, H., Boulanger, P., Le Lamer, S., Amighi, K. Preparation and in vitro/in vivo evaluation of nano-sized crystals for dissolution rate enhancement of ucb-35440-3, a highly dosed poorly water-soluble weak base. Europ J Pharm Biopharm, 64, 360-368, 2006.

27. Luo, Y., Chen, D., Ren, L., Zhao, X., Qin, J. Solid lipid nanoparticles for enhancing vinpocetine's oral bioavailability. J Control Release, 114, 53-59, 2006.

28. Barzegar-Jalali, M., Adibkia, K., Valizadeh, H., SiahiShadbad, M., Nokhodchi, A., Omidi, Y., Mohammadi, G., HallajNezhadi, S., Hasan, M. Kinetic analysis of drug release from nanoparticles. J Pharm Pharm Sci, 11, 167-177, 2008.

29. Mohammadi, G., Barzegar-Jalali, M., Valizadeh, H., Nazemiyeh, H., Barzegar-Jalali, A., Siahi Shadbad, MR., Adibkia, K., Zare, M. Reciprocal powered time model for release kinetic analysis of ibuprofen solid dispersions in oleaster powder, microcrystalline cellulose and crospovidone. J Pharm Pharm Sci, 13, 152-161, 2010. 